\title{
Tate Worlds
}

\section{Art and Artifacts Reimagined in Minecraft}

\author{
Eleanor Brooke Styles
}

Having captured imaginations worldwide, and with over 70 million in sales across platforms, the cultural power of Minecraft is not to be underestimated. Now the third best-selling game of all time, the sometimes explorative, sometimes creative, sandbox-style (i.e., unconstrained and free-roaming) video game presents many unique and exciting opportunities for facilitating audience-institution communication (Garrelts 2014). Based in an open world, Minecraft provides players with a virtual space within which they are free to explore and to create their own structures. Over the past few years, the educational possibilities of Minecraft for a younger audience have been increasingly realized and acted upon, as evidenced by the recent development of MinecraftEDU, an educational edition of the game for use within the classroom (Sáez-López et al. 2015). Less widely considered however, is the role it could play in heritage institutions, particularly for meaningfully engaging with a younger demographic. Some explorative steps have already been made by institutions such as the American Museum of Natural History, which made use of the educational edition to host online sessions for students on the themes of a 2013 exhibition about the production of food (Mooshme 2013). Similarly, Alaska's Anchorage Museum hosted an event in 2014 which saw players recreate the museum on a Minecraft server (Anchorage Museum 2014). This review will consider the use of Minecraft as a method of web-based communication, and as an experimental engagement technique, based on its application by the British Tate Galleries in their digital project 'Tate Worlds'. In looking primarily at how effective the Tate's use of Minecraft has been in reaching and communicating to its audience, this review will critically evaluate the use of the medium and the relationship between the dissemination of this project and its reception by users, as well as making recommendations for future heritage Minecraftbased projects (Figure 1).

The Tate is a group of four art galleries which house the national collection of British art, as well as sizable international, modern and contemporary collections. Their online presence is strong, with their principal Twitter account @tate, boasting the largest following of any art gallery in Europe (2.39 million) and the third greatest number of followers of an arts institution worldwide as of January 2016 (Tate 2015a). Tate's early uptake and application of both digital and social media places them in an enviable posi- tion amongst heritage institutions, as their reach and influence permeates far beyond the 7 million visitors that their physical gallery spaces receive annually (Tate 2015a).

As a project, the Minecraft-based Tate Worlds is shaped by Tate's mission statement 'to increase the public's knowledge, understanding and appreciation of art' (Tate 2015b), but it is also flavored by a willingness to experiment with newer technologies in order to achieve these goals. The project was conceived, designed and created in collaboration with freelance artist and advocate for the educational use of Minecraft, Adam Clarke (who acts as the Lead Artist and Producer for the project), alongside Multimedia Producer for Tate, Tony Guillan, and a small international team of map-makers. Tate Worlds was developed following Clarke's shortlisting for the 2014 IK prize at Tate Britain, which awards funding to projects which feature 'innovative applications of digital technology'(Tate 2015c).

The project consists of a series of downloadable Minecraft 'maps'-virtual environments populated by digital interpretations of artworks from Tate's collections, with narrative and interactive challenges inspired by the style and content of the original artwork. For example, a map inspired by Soul of the Soulless City, a Futurist-style painting by Christopher Nevinson, draws from the painting's prominent railway tracks and features a rollercoasterstyle ride through a recreated and sepia-toned 1920s New York (Figure 2). On the other hand, the Minecraft map for André Derain's Pool of London features highly saturated Fauvist colors and a quest to help the artist himself find his missing pigments (Tate 2016) (Figure 3).

Educational content about the works of art and their creators is disseminated through play via the use of in-game characters and written signs. These methods for facilitating learning are championed by supporters of Minecraft's educational use, as they allow players to enjoy the game without overly didactic messages. Minecraft is a platform ripe for engaging new and potentially younger audiences due to its very large and active online community, primarily on YouTube. Its sandbox format and ease of use make it ideal as a platform for creating and sharing content, with many players uploading videos of their adventures in-game to the site. As such, YouTube has become one of the 


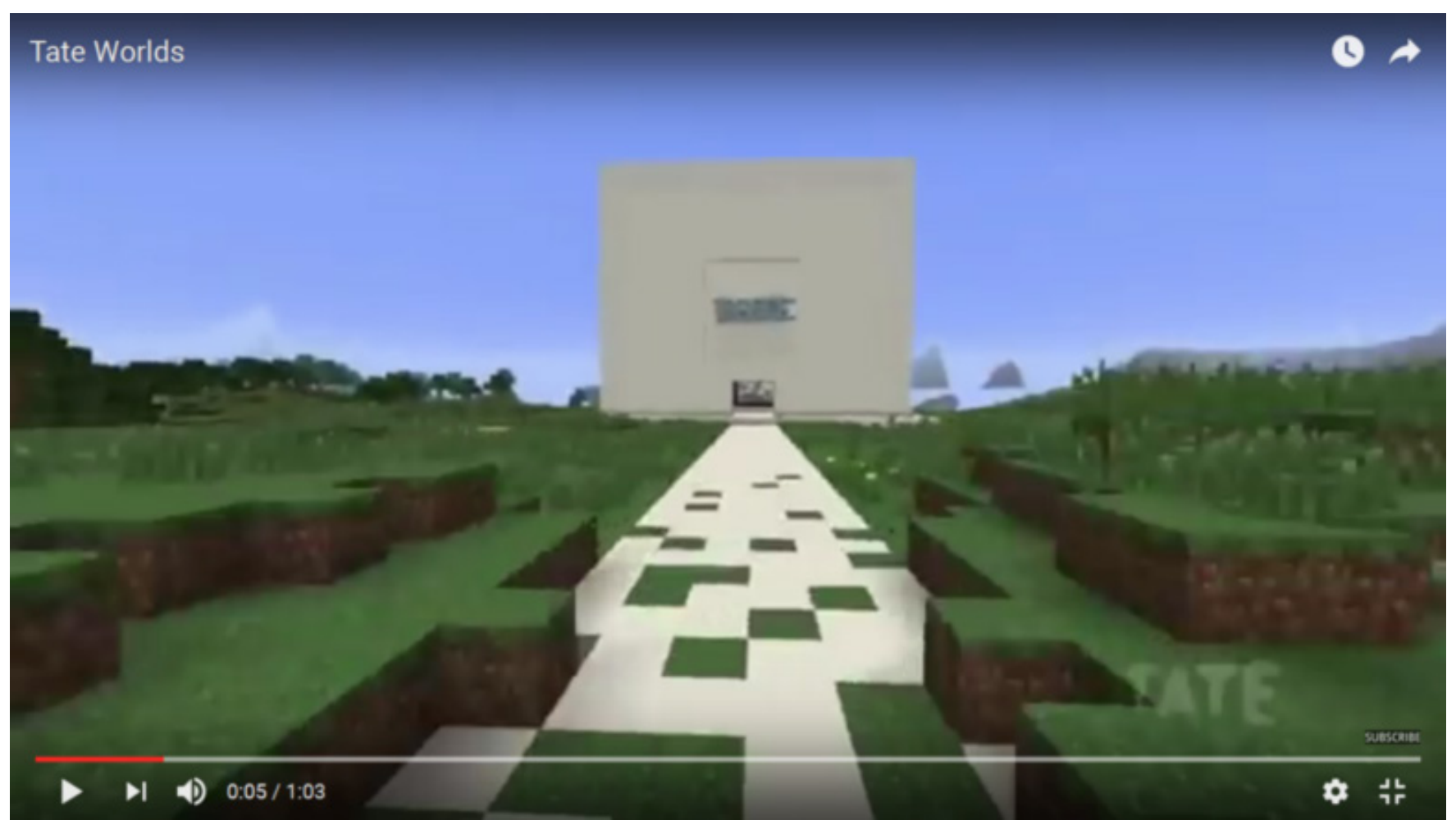

FIGURE 1. Tate's trailer for the Tate Worlds project https://youtu.be/OLsz1OZ9bpc
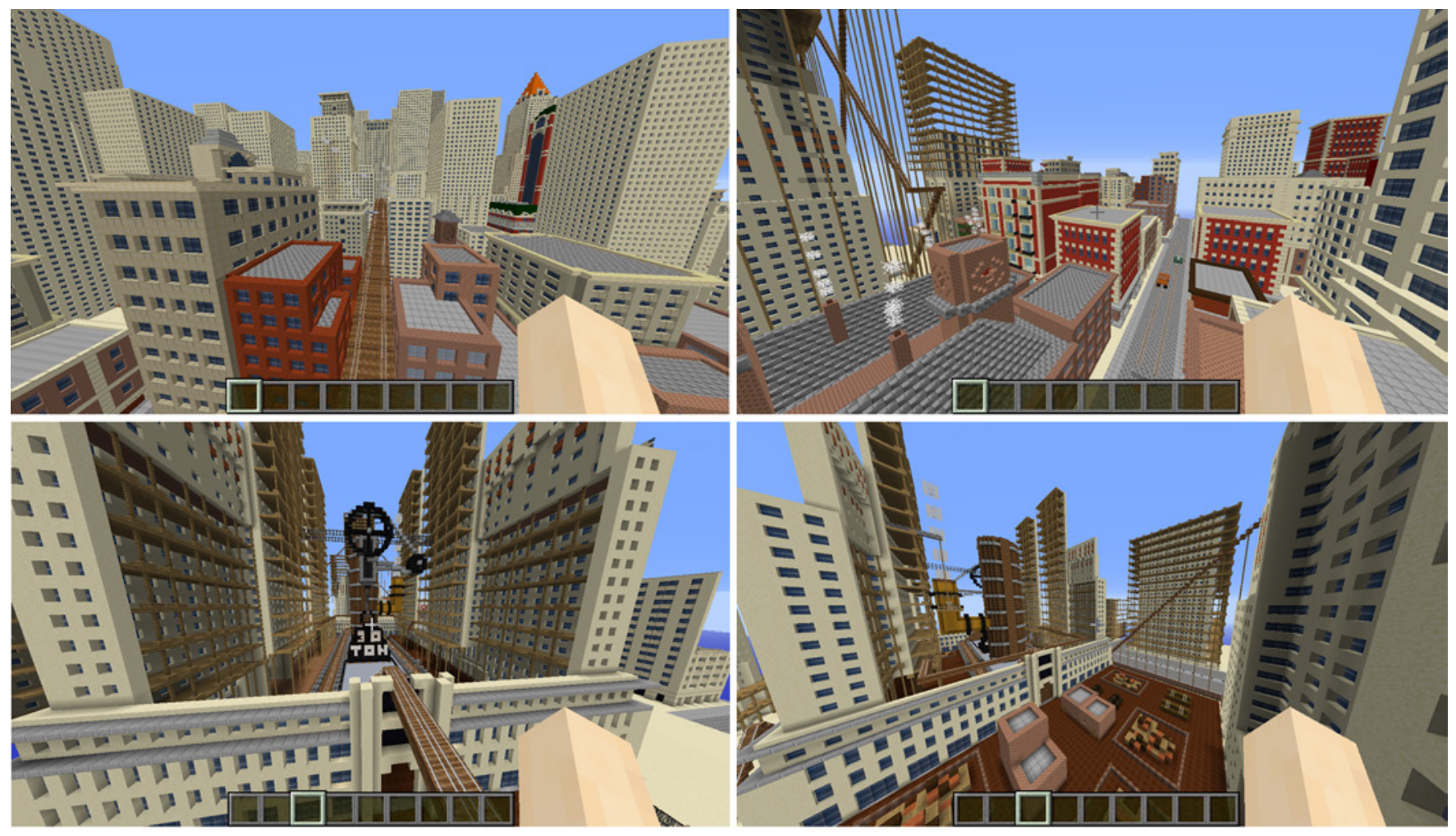

FIGURE 2. Screenshots of Minecraft maps based on the painting The Soul of the Soulless City. 


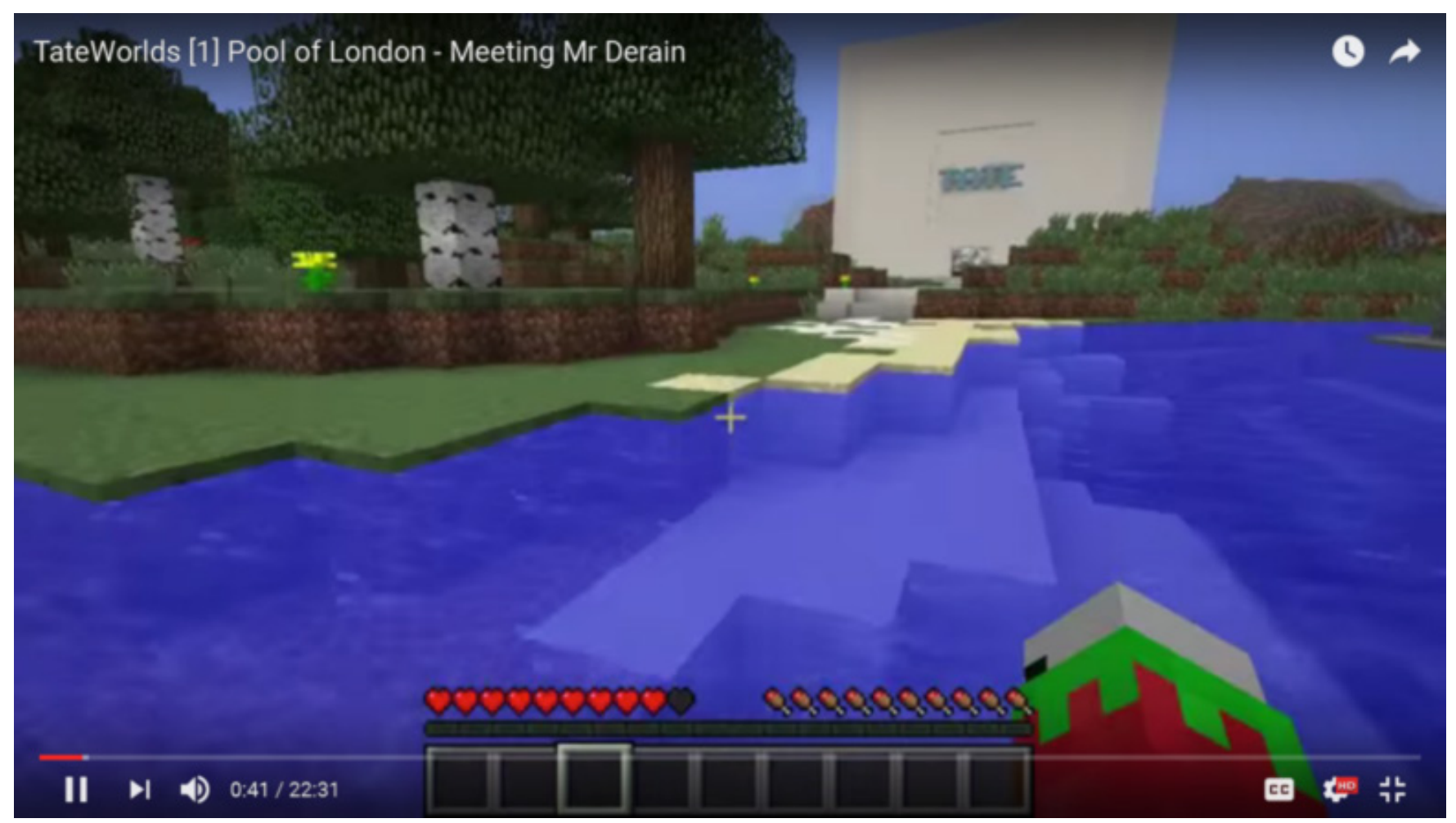

FIGURE 3. A youtube 'play-through' of The Pool of London Minecraft map by Adam Clarke (project leader) and his family https://youtu.be/o05ry4RnUcE

most popular ways in which a Minecraft map can gain traction, particularly if they have been played by one of a number of prominent YouTube Minecraft personalities. Unfortunately, there appears to be no such promotion for the Tate World maps. There are a number of smaller play-throughs and some behind the scenes videos released by the creators, but there appears to be no large traction for the project within the YouTube community. ${ }^{1}$ Attempts at engagement with larger YouTube personalities are evident, for example, in the presentation of the project at the UK's largest gaming convention Insomnia i53 in 2014. Here, Tate introduced the maps with the aid of popular British YouTuber and Minecraft player The Diamond Minecart. ${ }^{2}$ Similarly, one of the maps makes use of the voice of another British YouTuber, Stampylonghead. ${ }^{3}$ Crucially, however, despite involvement with the project, neither of these YouTubers have chosen to include a play through of the maps on their own YouTube pages. At best, this means that the Tate has unfortunately failed to gain the support of these popular YouTubers and their audiences. At worst, however, it potentially indicates that the Tate has either not understood the crucial role YouTube plays in supporting Minecraft, or, that players themselves do not consider the maps entertaining enough to play and publish online.

In either case, we must look at the ways in which the maps are instead being disseminated to audiences. The lack of meaningful engagement by Tate with the core of the YouTube Minecraft community is characteristic of what Russo and Peacock (2009) suggest is a 'build it and they will come' approach to outreach activities, with museums unintentionally over-valuing their outputs to the public. While maps can be downloaded directly from the Tate Worlds pages on Tate's website, there is limited signposting to these pages, making it difficult for the maps to reach intended audiences. Similarly, any mention of the project by other Tate-based media is limited. Tate most frequently tweets about Tate Worlds from the @Tatekids account, with tweets designed to encourage responses from the audience such as: "Have you found the fireworks in The Pool of London \#minecraft? Tweet your photo \#tateworlds" (Figure 4).

However, this makes two basic assumptions-firstly that their Minecraft audience is following this account, and secondly that their targeted audience is on Twitter at all. If, for a moment, we accept uncritically that the target audience for this project is 8-16 year olds, demographically, Twitter tends to appeal to an older audience than Minecraft, and certainly the youngest of the target audience would be unlikely to be using Twitter at all. There are also questions over the target audience's interest in traditional artwork, and their likelihood to either follow the Tate accounts or to play the produced maps based on their content. Potentially of course, Tate's Twitter offering may be designed for parents to find the project and suggest it to their children, but if so, the tweets are addressing the wrong audience. This is reflected in the lack of replies to these tweets and the limited engagement on this platform. This is not to say, however, that the project is not being accessed by the community, or that it has been entirely unsuccessful in reaching intended audiences. The Tate Worlds maps have also been posted on www. minecraftmaps.com; an online community repository for maps. As of the end of January 2016, the total number of downloads across the three maps currently available from this site amounted to 
T̃TE Tate Kids KIDS @tate_kids Have you found the fireworks in The Pool of
London \#minecraft? Tweet your photo
\#tateworlds ow.ly/lpOAG

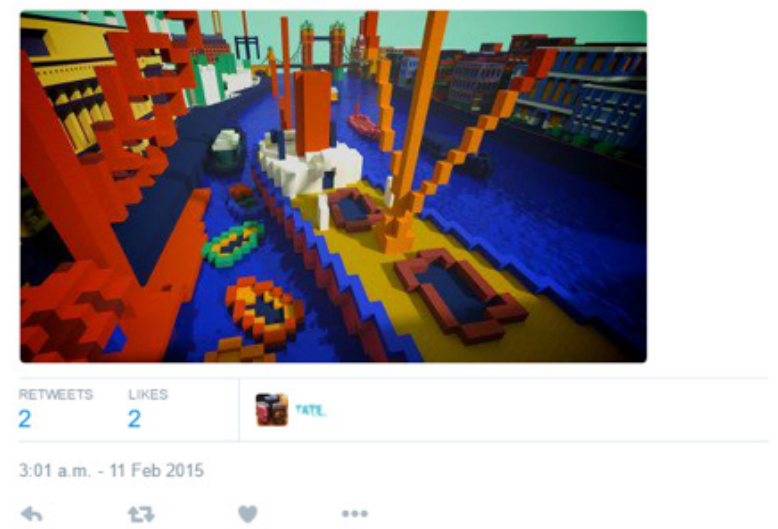

FIGURE 4. Tweet from the @tatekids account regarding the Tateworlds project https://twitter.com/tate kids/ status/565465642938433536

190,120 (minecraftmaps.com 2016). ${ }^{4}$ These figures combine to represent a significant engagement with the maps, particularly without a large amount of work perceivably being done to promote them by Tate.

However, while they may be reaching their audiences, it is another question entirely if these individuals are meaningfully and effectively engaging with them. The only data available in this respect (due to the lack of response by the audience on any platform curated by the Tate), are comments made in May 2015 by Tony Guillan, who, speaking for Tate, claimed that the project had already been 'hugely successful' in increasing appeal and visits amongst young audiences coming to see the real art the project's Minecraft maps were based on (Blooloop 2014). Interestingly, this reflects the work of Newell (2012) who suggests that despite, or perhaps because of, a growing digitization of objects and the new digital media interpretations that these can inspire, the original artifact itself remains important to people. The maps can therefore be considered as additions to the physical galleries which can deepen the relationship the audience has with an artifact or site. It is perhaps too soon to measure the overall success of the project in this way due to its ongoing status, but if we simply examine the statistics of downloaded maps, at least latently the information which the project incorporates into the map's narrative will build an understanding and recognition of artworks and their artists.

However, while this is undoubtedly a positive outcome of the project, there remains a major issue with its implementation. While their decision to engage a younger audience through use of the platform was a strong one, crucially, Tate are not using the Minecraft platform to its full potential. The largest issue is the fact that they have failed to harness and utilize aspects of creative collaboration, which Minecraft offers in abundance. As a result, these maps, while based on a principle of interactivity with art, fail to facilitate any kind of direct visitor-institution communication or interaction. Within the framework of engagement online set out by Kidd $(2011,71)$, this level of communication falls short of the collaborative standards which the Tate seems to want to emulate. Instead, it follows and enforces the established dichotomy of roles, with the museum as the body of authority, providing content to and for its audience, without opportunity for the audience to co-produce and share ideas (Simon 2010). Tate could take the project one step further and attempt to relinquish, at least partially, this role of authority and in doing so build a stronger platform of engagement. Online servers, which allow creative, free-builds, are exceptionally popular within the Minecraft community, as are 'tours' of these servers. It would not be beyond the capabilities of the Tate to host and provide the necessary moderation needed for such worlds. Here, using suggestions and inspiration provided by the Tate and their collection of work, players could actively participate by creating new art-themed structures and builds. This creative exploration of art via Minecraft could also take place offline, if a means for sharing creations was suitably developed. At the very least, audience-submitted builds could be featured weekly on the Tate's media, perhaps via the Tate Kids platform, linking it to the project via the use of hashtags, and therein strengthening the links between the museum and its audiences, as well as increasing the response and recognition of the project.

In summary, limitations aside, the Tate Worlds project can be regarded as an admirable and forward-thinking attempt at integrating digital heritage within non-digital galleries. While the Tate's efforts may lack the necessary online support required to attract a wealth of players in an already over-saturated environment, the headway they have made into Minecraft's application within heritage is undeniable. If future projects were to draw inspiration from Tate's approach, the largest take-away from the project should be the use of Minecraft as a web-based communication medium, fully capable and appropriate for connecting institutions to younger audiences. This creation of a virtual learning environment in Minecraft is an interpretive activity which can easily be implemented at a number of arts and heritage sites, with a wide variety of outcomes. In particular, the concept of cocreation that Minecraft can facilitate may offer up many benefits. While there are clear limitations to Minecraft's use, such as the fact that much of the communication created must be linked to other platforms such as YouTube, Twitter or Minecraft forums, Tate Worlds demonstrates that its use can be successful in driving visits and in engaging with wider audiences.

\section{REFERENCES CITED}

\section{Anchorage Museum}

2016 Minecraft Members Build Museum, One Brick at a Time. Electronic document, https://www.anchoragemuseum.org/about-us/news/ community-archive/minecraft-members-build-museum-one-brick-at-atime, accessed April 4, 2016.

Blooloop

2016 The Genius of Tate Worlds in Minecraft. Electronic document, http://www.blooloop.com/features/the-genius-of-tate-worlds-inminecraft/32745\#.VqYA4yqLTIU, accessed January 25, 2016.

Clarke, Adam

2016 Tate Worlds Launched at Insomnia Gaming Festival i53. Electronic document, http://thecommonpeople.tv/tate-worlds-launched-atinsomnia-gaming-festival-i53, accessed January 27, 2016. 
Garrelts, Nate

2014 Understanding Minecraft. Essays on Play, Community and Possibilities. McFarland, Jefferson.

Giaccardi, Elisa

2012 Heritage and Social Media: Understanding Heritage in a Participatory Culture. Routledge, London.

Kalay, Yehuda E., Thomas Kvan, and Janice Affleck

2008 New Heritage: New Media and Cultural Heritage. Routledge, London.

Kidd, Jenny

2011 Enacting Engagement Online: Framing Social Media Use for the Museum. Information Technology \& People 24(1):64-77.

Mooshme

2016 The Launch of Minecraft at the Museum of Natural History. Electronic document, http://www.mooshme.org/2013/09/the-launch-ofminecraft-at-the-museum-of-natural-history/, accessed April 4, 2016

Newell, Jenny

2012 Old Objects, New Media: Historical Collections, Digitization and Affect. Journal of Material Culture 17:287-306.

Russo, Angelina and Darren Peacock

2009 Great Expectations: Sustaining Participation in Social Media Spaces. Electronic document, http://www.museumsandtheweb.com/mw2009/ papers/russo/russo.html, accessed January 25, 2016.

Sáez-López, José-Manuel, John Miller, Esteban Vázquez-Cano, and MaríaConcepción Domínguez-Garrido

2015 Exploring Application, Attitudes and Integration of Video Games: MinecraftEdu in Middle School. Journal of Educational Technology \& Society 18: 114-128.

Simon, Nina

2010 The Participatory Museum. Museum 2.0, Santa Cruz.

Socialblade.com,

2016 YouTube Stats, Channel Statistics - Socialblade.com. Electronic document, http://socialblade.com/youtube/, accessed January 25, 2016.

Tate

2015a Tate Annual Report 2014/15. Electronic document, http://www.tate. org.uk/download/file/fid/44097, accessed January 27, 2016

2015b Tate Mission Statement. Electronic document, http://www.tate.org. uk/about/our-work/collection/acquisitions, accessed January 27, 2016.

2015c Tate IK Prize. Electronic document, www.tate.org.uk/about/projects/ ik-prize, accessed January 27, 2016.

2016 Tate Worlds. Electronic document, http://www.tate.org.uk/about/ projects/tate-worlds-art-reimagined-minecraft, accessed January 27, 2016.

\section{NOTES}

1. A search for 'tateworlds' on YouTube turns up 1,270 unique videos, the most popular of which has just under 12,000 views. The cumulative view count for a series of five videos released by Dragnoz on the creation of the map represent roughly 148,000 views. While impressive, these figures represent only a limited engagement with the maps, particularly when popular maps played by YouTube personalities frequently have the potential to gain in excess of a million views.

2. As of the end of January 2016. The Diamond Minecart's channel had $9,229,528$ subscribers and 5,601,858,623, with YouTube analytics site socialblade.com placing him nineteenth in the world for view-count and sixtieth for subscriber count worldwide (socialblade.com, 2016)

3. Representing $6,967,587$ subscribers and 4,603,201,890 views, Stampylonghead has a slightly lower - yet still substantive - view rank of 35 (in comparison to The Diamond Minecart) and subscriber rank of 108 (socialblade.com, 2016)

4. Unfortunately this cannot be compared or added to the number of downloads from the Tate site as these figures are not made available to the public, nor does it take into account the two Tate World maps hosted online which do not require downloading. At least one of these frequently records around 40,000 unique visitors per week, due to its inclusion and easy access from popular Minecraft server network Hypixel. 